\title{
Multiscale Matrix Sampling and Sublinear-Time PageRank Computation
}

Christian Borgs, Michael Brautbar, Jennifer Chayes, and Shang-Hua Teng

Abstract. A fundamental problem arising in many applications in Web science and social network analysis is the problem of identifying all nodes in a network whose PageRank exceeds a given threshold $\Delta$. In this paper, we study the probabilistic version of the problem whereby given an arbitrary approximation factor $c>1$, we are asked to output a set $S$ of nodes such that with high probability, $S$ contains all nodes of PageRank at least $\Delta$, and no node of PageRank smaller than $\Delta / c$. We call this problem SignificantPageRAnKs.

We develop a nearly optimal local algorithm for the problem with time complexity $\tilde{O}(n / \Delta)$ on networks with $n$ nodes, where the tilde hides a polylogarithmic factor. We show that every algorithm for solving this problem must have running time of $\Omega(n / \Delta)$, rendering our algorithm optimal up to logarithmic factors. Our algorithm has sublinear time complexity for applications including Web crawling and Web search that require efficient identification of nodes whose PageRanks are above a threshold $\Delta=n^{\delta}$, for some constant $0<\delta<1$.

Our algorithm comes with two main technical contributions. The first is a multiscale sampling scheme for a basic matrix problem that could be of interest on its own. For us, it appears as an abstraction of a subproblem we need to tackle in order to solve the

Color versions of one or more of the figures in the article can be found online at www.tandfonline.com/uinm. 
SignificantPageRANKS problem, but we hope that this abstraction will be useful in designing fast algorithms for identifying nodes that are significant beyond PageRank measurements.

In the abstract matrix problem, it is assumed that one can access an unknown rightstochastic matrix by querying its rows, where the cost of a query and the accuracy of the answers depend on a precision parameter $\epsilon$. At a cost propositional to $1 / \epsilon$, the query will return a list of $O(1 / \epsilon)$ entries and their indices that provide an $\epsilon$-precision approximation of the row. Our task is to find a set that contains all columns whose sum is at least $\Delta$ and omits every column whose sum is less than $\Delta / c$. Our multiscale sampling scheme solves this problem with cost $\tilde{O}(n / \Delta)$, while traditional sampling algorithms would take time $\Theta\left((n / \Delta)^{2}\right)$.

Our second main technical contribution is a new local algorithm for approximating personalized PageRank, which is more robust than the earlier ones developed in [Jeh and Widom 03, Andersen et al. 06] and is highly efficient, particularly for networks with large in-degrees or out-degrees.

Together with our multiscale sampling scheme, we are able to solve the SignificanTPAGERANKS problem optimally.

\section{Introduction}

A basic problem in network analysis is to identify the set of network nodes that are "significant." For example, they could be the significant Web pages that provide the authoritative contents in a Web search; they could be the critical proteins in a protein interaction network; and they could be the set of people (in a social network) most influential in seeding online advertising. As networks become larger, we need more efficient algorithms to identify these "significant" nodes.

\section{I.I. Identifying Nodes with Significant PageRanks}

The meanings and measures of significant vertices depend on the semantics of the network and on the proposed applications. In this paper, we focus on a particular measure of significance: the PageRank of the vertices.

Formally, the PageRank (with restart constant $\alpha$, also known as the teleportation constant) of a Web page is proportional to the probability that the page is visited by a random surfer who explores the Web using the following simple random walk: at each step, go with probability $(1-\alpha)$ to a random Web page linked from the current page, and with probability $\alpha$, restart the process from a uniformly chosen Web page. For ease of presentation of our later results, we consider a normalization of PageRank so that the sum of the PageRank values over all vertices is equal to $n$, the number of vertices in the network:

$$
\sum_{u \in V} \operatorname{PageRank}(u)=n \text {. }
$$


PageRank has been used by the Google search engine and has found applications in a wide range of data analysis problems [Berkhin 05, Brin and Page 98]. In this paper, we consider the following natural problem of finding vertices with "significant" PageRank.

Problem I.I. (SignificantPageRAnks.) Given a network $G=(V, E)$, a threshold value $1 \leq \Delta \leq|V|$, and a positive constant $c>1$, compute a subset $S \subseteq V$ with the property that $S$ contains all vertices of PageRank at least $\Delta$ and no vertex of PageRank less than $\Delta / c$.

For the corresponding algorithmic problem, we assume that the network topology is described in the sparse representation of an (arbitrarily ordered) adjacency list for each vertex, as is natural for sparse graphs such as social and information networks. We are interested in developing an efficient local algorithm [Spielman and Teng 13, Andersen et al. 06, Andersen et al. 08] for the problem in the context of Web applications. The algorithm is allowed to sample randomly only out-links of previously accessed nodes in addition to sampling nodes uniformly at random from the network. This model is highly suitable for PageRank maintenance in Web graphs and online information networks.

As the main contribution of this paper, we present a nearly optimal local algorithm for SignificAntPAGERANKS.

The running time of our algorithm is $\tilde{O}(n / \Delta)$. We also show that every algorithm for SignificAnTPAgERANKS must have query complexity as well as runtime complexity $\Omega(n / \Delta)$. Thus, our algorithm is optimal up to a logarithmic factor. Note that when $\Delta=\Omega\left(n^{\delta}\right)$, for some constant $0<\delta<0$, our algorithm has sublinear time complexity.

Our SignificantPageRAnKs algorithm applies a multiscale matrix sampling scheme that uses a fast personalized PageRank estimator (see below) as its main subroutine.

\section{I.2. Personalized PageRanks}

While the PageRank of a vertex captures the importance of the vertex as collectively assigned by all vertices in the network, one can use the distributions of the following random walk to define the pairwise contributions of significance [Haveliwala 03]: given a teleportation probability $\alpha$ and a starting vertex $u$ in a network $G=(V, E)$, go at each step with probability $(1-\alpha)$ to a random neighboring vertex, and with probability $\alpha$, restart the process from $u$. For $v \in V$, the probability that $v$ is visited by this random process, denoted by PersonalizedPageRank $\operatorname{Pa}_{u}(v)$, is $u$ 's personal PageRank contribution of significance to $v$. It is not hard to verify 
that

$$
\begin{array}{ll}
\forall u \in V, & \sum_{v \in V} \operatorname{PersonalizedPageRank}_{u}(v)=1, \\
\forall v \in V, & \operatorname{PageRank}(v)=\sum_{u \in V} \operatorname{PersonalizedPageRank}_{u}(v) .
\end{array}
$$

Personalized PageRanks have been widely used to describe personalized behavior of Web users [Page et al. 98] as well as for developing good network clustering techniques [Andersen et al. 06]. As a result, fast algorithms for computing or approximating personalized PageRank are quite useful. One can approximate PageRanks and personalized PageRanks by the power method [Berkhin 05], which involves costly matrix-vector multiplications for large-scale networks. Applying effective truncation, [Jeh and Widom 03] and [Andersen et al. 06] developed personalized PageRank approximation algorithms that can find an $\epsilon$-additive approximation in time proportional to the product of $\epsilon^{-1}$ and the maximum in-degree in the graph.

\section{I.3. Multiscale Matrix Sampling}

Following the matrix view of the personalized PageRank formulation of [Haveliwala 03] and the subsequent approximation of algorithms in [Jeh and Widom 03, Andersen et al. 06], we introduce a matrix problem whose solution would lead to fast PageRank approximation and sublinear-time algorithms for SignificANTPageRAnks.

In the basic form of this matrix problem, we consider a black-box model for accessing an unknown $n \times n$ right-stochastic matrix, in which we can make a query only of the form matrixAccess $(i, \epsilon)$, where $1 \leq i \leq n$ and $\epsilon \in(0,1]$. This query will return, with high probability, a list of $O(1 / \epsilon)$ entry-index pairs that provide an $\epsilon$-precise approximation of row $i$ in the unknown matrix: for each $1 \leq j \leq n$, if $(p, j)$ is in the list of entry-index pairs returned by matrixAccess $(i, \epsilon)$, then $\left|p-m_{i, j}\right| \leq \epsilon$, where $m_{i, j}$ is the $(i, j)$ entry of the unknown matrix; otherwise, if there is no entry containing index $j$, then $m_{i, j}$ is guaranteed to be at most $\epsilon$. Further, the cost of this query is propositional to $1 / \epsilon$. We will refer to this blackbox model as the sparse and approximate row access model, or SARA model for short.

We now define the basic form of our matrix problem:

Problem I.2. (SignificantMatrixColumns.) Given an $n \times n$ right-stochastic matrix $M$ in the SARA model, a threshold $\Delta$, and a positive constant $c>1$, return a subset of columns $S \subseteq V$ with the property that $S$ contains all columns of sum at least $\Delta$ and no column with sum less than $\Delta / c$. 
There is a straightforward connection between SignificantMatrixColumns and SignificantPageRAnks. Following [Andersen et al. 08], we define a matrix PPR (short for PersonalizedPageRank) to be the $n \times n$ matrix whose $u$ th row is

\section{PersonalizedPageRank $_{u}(\cdot)$.}

Clearly, PPR is a right-stochastic matrix, and for $1 \leq v \leq n$, $\operatorname{PageRank}(v)$ is equal to the sum of the $v$ th column in PPR. Therefore, if we can solve the SignificantMatrixColumns problem with cost $\tilde{O}(n / \Delta)$ and also solve the problem of computing an $\epsilon$-additive approximation of personalized PageRank in $\tilde{O}(\log (n) / \epsilon)$ time, then we shall be able to solve SignificantPageRAnks in $\tilde{O}(n / \Delta)$ time.

In this paper, we analyze a multiscale sampling algorithm for SigNIFICANTMATRIXCOLumns. The algorithm selects a set of precision parameters $\left\{\epsilon_{1}, \ldots, \epsilon_{h}\right\}$, where $h$ grows linearly with $n / \Delta$ and $\epsilon_{i}=i / h$. It then makes use of sparse-andapproximate-row-access queries to obtain approximations of randomly sampled rows. For each $i$ in the range $1 \leq i \leq h$, the algorithm makes $\tilde{O}(1)$ (depending on the desired success probability) row-access queries to get a good approximation of the contribution of column elements of value of order $\epsilon_{i}$. We show that with probability $1-o(1)$, the multiscale sampling scheme solves SignificanTMATRIXCOLumns with cost $\tilde{O}(n / \Delta)$.

While we could present our algorithm directly on PPR, we hope that this matrix abstraction will enable us to highlight the two key algorithmic components in our fast PageRank approximation algorithm: multiscale sampling and robust approximation of personalized PageRanks.

\section{I.4. Robust Approximation of PersonalizedPageRanks}

For networks with constant maximum degrees, we can simply use personalized PageRank approximation algorithms developed in [Jeh and Widom 03] or [Andersen et al. 06] inside the multiscale scheme to obtain an $\tilde{O}(n / \Delta)$-time algorithm for SignificantPageRAnks. However, for networks such as Web graphs and social networks that may have nodes with large degrees, these two approaches are insufficient for our needs.

We develop a new local algorithm for approximating personalized PageRank that satisfies the desirable robustness property that our multiscale sample scheme requires. Given $\lambda, \epsilon>0$ and a starting vertex $u$ in a network $G=(V, E)$, our algorithm estimates each entry in the personalized PageRank vector defined by $u$,

PersonalizedPageRank $(u, \cdot)$, 
to a $[1-\lambda, 1+\lambda]$ multiplicative approximation around its value plus an additive error of at most $\epsilon$. The time complexity of our algorithm is

$$
O\left(\frac{\log ^{2} n \log \left(\epsilon^{-1}\right)}{\epsilon \lambda^{2}}\right) .
$$

Our algorithm requires a careful simulation of random walks from the starting node $u$ to ensure that its complexity does not depend on the degree of any node. Together with the multiscale sampling scheme, this algorithm leads to an $\tilde{O}(n / \Delta)$-time algorithm for SignificAntPAGERANKS. We conclude our analysis by showing that our algorithm for solving SignificantPageRAnks is optimal up to a polylogarithmic factor.

Remark 1.3. While the main contribution of this paper is theoretical, that is, our focus is to design the first nearly optimal local algorithm for PageRank approximation, we hope that our algorithm or its refinements can be useful in practical settings for analyzing large-scale networks. For example, our sublinear algorithm for SignificantPageRAnks could be used in Web search engines, which often need to build a core of Web pages, to be used later for Web search. It is desirable that pages in the core have high PageRank values. These search engines usually apply crawling to discover new significant pages and insert them into the core to replace existing core pages with relatively low PageRank values. As noted already, our algorithms are local and are implementable in various network querying models that assume no direct global access to the network but allow one to generate random out-links of a given node as well as to sample nodes from the network uniformly at random. Such an implementation is desirable for processing large social and information networks as in the construction of the core pages for Web search. We also anticipate that our algorithm for SignifiCANTPAGERANKS and the multiscale scheme for its matrix abstraction will be useful for many other network analysis tasks.

\section{I.5. Related Work}

Our research is inspired by the body of work on local algorithms [Spielman and Teng 13, Andersen et al. 06, Andersen et al. 08], sublinear-time algorithms [Rubinfeld and Shapira 11], and property testing [Goldreich 10], which study algorithm design for finding relevant substructures or estimating various quantities of interest without examining the entire input. Particularly, we focus on identifying nodes with significant PageRanks and approximating personalized PageRanks without exploring the entire input network. In addition, our framework is based on a combination of uniform crawling and uniform sampling of vertices in 
a graph, and hence it can be viewed as a sublinear algorithm (when $\Delta=n^{\Omega(1)}$ ) in a rather general access model as discussed in [Rubinfeld and Shapira 11].

It is well known that in a directed graph, high in-degree of a node does not imply high PageRank for that node and vice versa. In fact, even in real-world Web graphs, only weak correlations have been reported between PageRank and in-degree [Pandurangan et al. 06]. One therefore needs to use methods for PageRank estimation that are not based solely on finding high in-degree nodes. Indeed, over the past decade, various beautiful methods have been developed to approximate the PageRank of all nodes. The common thread is that they all run in time at least linear in the input (see [Berkhin 05] for a survey of results). Perhaps closest to our framework are the following two Monte Carlo-based approaches: The PageRank estimation method of [Avrachenkov 07] conducts simulation of a constant number of random walks from each of the nodes in the network, and therefore it requires linear time in the size of the network. A similar approach is analyzed in [Bahmani et al. 10], where a small number of random walks are computed from each network node, which shows that a tight estimate for the PageRank of a node with a large enough PageRank can be computed from the summary statistics of these walks. In addition, the paper shows how these estimates can be kept up to date, with a logarithmic factor overhead, on a certain type of a dynamic graph in which a fixed set of edges is inserted in a random order.

Our scheme is suitable for any network with arbitrary changes in it as well, including addition or removal of edges and nodes, with the necessary computation being performed "on the fly" as needed. But in contrast to the above approaches, for $\Delta=n^{\Omega(1)}$, our construction gives a sublinear-time algorithm for identifying all nodes whose PageRanks are above the threshold $\Delta$ and approximating their PageRanks.

We have benefited from the intuition of several previous works on personalized PageRank approximation. Jeh and Widom developed a method based on a deterministic simulation of random walks by pushing out units of mass across nodes [Jeh and Widom 03]. Their algorithm gives an $\epsilon$-additive approximation with runtime cost of order of $\log n / \epsilon$ times the maximum out-degree of a node in the network. Andersen, Chung, and Lang provided a clever implementation of the approach of Jeh and Widom that removes the $\log n$ factor from the runtime cost, still stopping when the residual amount to push out per node is at most $\epsilon$ [Andersen et al. 06]. ${ }^{1}$ We note, however, that for networks with large out-degrees, the complexity of this algorithm may not be sublinear.

\footnotetext{
${ }^{1}$ Thus at termination, the infinity norm of the residual vector is at most $\epsilon$, which can easily be shown to bound from above the infinity norm of the difference between the true personalized PageRank vector and the estimation computed.
} 
In [Andersen et al. 08], a "backward-running" version of the local algorithm of [Andersen et al. 06] was developed. That algorithm finds an $\epsilon$-additive approximation to the PageRank vector with running time proportional to the product of $1 / \epsilon$, the maximum in-degree in the network, and the PageRank value. The authors show how it can be used to provide some reliable estimate to a node's PageRank: for a given $k$, with running time proportional to $\tilde{\Theta}(k)$ times the maximum in-degree in the network (and no dependency on the PageRank value), it can bound the total contribution from the $k$ highest contributors to a given node's PageRank. However, for networks with large in-degrees, its complexity may not be sublinear even for small values of $k$. We also note that the method does not scale well for estimating the PageRank values of multiple nodes, and the algorithm needs to be run separately for each target node.

The problem of SignificantMatrixColumns can also be viewed as a matrix sparsification or matrix approximation problem, where the objective is to remove all columns with $l_{1}$ norm less than $\Delta / c$ while keeping all columns with $l_{1}$ norm at least $\Delta$. To achieve time efficiency, it is essential to allow the algorithm the freedom in deciding whether to keep or delete columns whose $l_{1}$ norm is in the range $[\Delta / c, \Delta]$.

While there has been a large body of work on finding a low-complexity approximation to a matrix (such as a low-rank matrix) that preserves some desirable properties, many of the techniques developed are not directly applicable to our task.

First, we would like our algorithms to work even if the graph does not have a good low-rank approximation; indeed, all of our algorithms work for any input graph. Second, our requirement to approximately preserve the $l_{1}$ norm only for significant columns enables us to achieve $\tilde{O}(n / \Delta)$ complexity for every stochastic matrix, whereas all low-rank matrix approximations run in time at least linear in the number of rows and columns of the matrix in order to reconstruct a low-rank approximation explicitly; see [Kannan 10, Kannan and Vempala 09] for recent surveys on low-rank approximations.

At a high level, the problem of SignificantMatrixColumns may seem to share some resemblance to the heavy-hitters problem considered in the datastreaming literature [Cormode and Muthukrishnan 05]. In the heavy-hitter problems, the goal is to identify all elements in a vector stream that have value greater than the sum of all elements. The main difficulty to overcome is the sequential order by which items arrive and the small space one can use to store information about them. The main technique used to overcome these difficulties is the use of multiple hash functions, which allows for concise summary of the frequent items in the stream. However, in SignificantMatrixColumns, we are faced with a completely different type of constraint - access to only a small fraction 
of the input matrix (in order to achieve sublinear running time) and having a precision-dependent cost of matrix row approximations. As a result, hashing does not seem to be a useful avenue for this goal, and one needs to develop different techniques in order to solve the problem.

\subsection{Organization}

In Section 2, we introduce some notation that will be used in the remainder of the paper. In Section 3, to better illustrate the multiscale framework, we present a solution to a somewhat simpler abstract problem that distills the computational task we use to solve SignificantMatrixColumns. In particular, we consider a black-box model accessing an unknown vector that either returns an exact answer or 0. Like the access model in SignificantMatrixColumns, higher precision costs more. In Section 4, we present our multiscale sampling algorithm for SignificantMatrixColumns. In Section 5, we address the problem of finding significant columns in a PageRank matrix by giving a robust local algorithm for approximating personalized PageRank vectors. The section ends with a presentation of a tight lower bound for the cost of solving SignificantMatrixCoLumns over PageRank matrices.

\section{Preliminaries}

In this section, we introduce some basic notation that we will use frequently. For a positive integer $n,[1: n]$ denotes the set of all integers $j$ such that $1 \leq j \leq n$. If $M \in \mathbb{R}^{n \times n}$ is an $n \times n$ real matrix, then for $v \in[1: n]$, we will use $M(v, \cdot)$ and $M(\cdot, v)$ to denote the $v$ th row and the $v$ th column of $M$, respectively. We denote the sum of column $v$ in $M$ by ColumnSum $(M, v)$. When the context is clear, we shall suppress $M$ in this notation and denote it by $\operatorname{ColumnSum}(v)$.

Most graphs considered in this paper are directed. For a given directed graph $G=(V, E)$, we usually assume $V=[1: n]$. We use an $n \times n$ matrix $A(G)$ to denote the adjacency matrix of $G$. In other words,

$$
A(i, j)= \begin{cases}1 & \text { if }(i, j) \in E \\ 0 & \text { otherwise }\end{cases}
$$

The PageRank vector of a graph $G$ is the (unique) stationary point of the following equation [Page et al. 98, Haveliwala 03]:

$$
\operatorname{PageRank}(\cdot)=\alpha \cdot \mathbf{1}^{n}+(1-\alpha) \operatorname{PageRank}(\cdot) \cdot D^{-1} A(G),
$$


where $\mathbf{1}^{n}$ is the $n$-place row vector of all 1 's, $0<\alpha<1$ is a teleportation probability constant, and $D$ is a diagonal matrix with the out-degree of $v$ at entry $(v, v)$.

Similarly, the personalized PageRank vector of $u$ in the graph $G$ is the (unique) stationary point of the following equation [Haveliwala 03]:

$\operatorname{PersonalizedPageRank}_{u}(\cdot)=\alpha \cdot \mathbf{1}_{u}+(1-\alpha) \operatorname{PersonalizedPageRank}_{u}(\cdot)$ $\cdot D^{-1} A(G)$,

where $\mathbf{1}_{u}$ is the indicator function of $u$.

Note that with the above definition of PageRank, the sum of the entries of the PageRank vector is normalized to $n$. This normalization is more natural in the context of personalized PageRank than the traditional normalization in which the sum of all PageRank entries is 1 .

For every $x, \log (x)$ means $\log _{2}(x)$ and $\ln (x)$ denotes the natural logarithm of $x$.

\section{Multiscale Approximation of Vector Sum}

Before presenting our algorithms for SignificantMatrixColumns, we give a multiscale algorithm for a much simpler problem that captures, we hope, the essence of the general algorithm.

We consider the following black-box model for accessing an unknown vector $\mathbf{p}=\left(p_{1}, \ldots, p_{n}\right) \in[0,1]^{n}$ : We can access the entries of $\mathbf{p}$ only by making a query of the form vector $\operatorname{Access}(i, \epsilon)$. If $p_{i} \geq \epsilon$, the query $\operatorname{vector} \operatorname{Access}(i, \epsilon)$ returns $p_{i}$; otherwise, when $p_{i}<\epsilon$, vector $\operatorname{Access}(i, \epsilon)$ returns 0. Furthermore, vectorAccess $(i, \epsilon)$ incurs a cost of $1 / \epsilon$. In this subsection, we consider the following abstract problem.

Problem 3.I. (VECTORSuM.) Given a black-box model vectorAccess $(\cdot)$ for accessing an unknown vector $\mathbf{p}=\left(p_{1}, \ldots, p_{n}\right) \in[0,1]^{n}$, a threshold $\Delta \in[1: n]$, and a positive constant $c>1$, return PASS if $\sum_{i} p_{i} \geq \Delta$, return FAIL if $\sum_{i} p_{i}<\Delta / c$, and otherwise return either FAIL or PASs.

To motivate our approach, before describing our multiscale algorithm to solve this problem, let us first analyze the running time of a standard sampling algorithm. In such an algorithm, one would take $h$ i.i.d. samples $s_{1}, \ldots, s_{h}$ uniformly from $[1: n]$ and query $p_{s_{t}}$ at some precision $\epsilon$ to obtain an estimator

$$
\frac{n}{h} \sum_{t=1}^{h} p_{s_{t}} \mathbf{I}\left[p_{s_{t}} \geq \epsilon\right]
$$


for the sum $\sum_{i} p_{i}$. The error stemming from querying at precision $\epsilon$ would be of order $n \epsilon$, so we clearly will have to choose $\epsilon$ of order $\Delta / n$ or smaller not to drown our estimate in the query error, leading to a running time of order $h n / \Delta$. The number of samples $h$, on the other hand, has to be large enough to guarantee concentration, which at a minimum requires that the expectation of the sum $\sum_{t=1}^{h} p_{s_{t}} \mathbf{I}\left[p_{s_{t}} \geq \epsilon\right]$ be of order at least unity. But the expectation of this sum is bounded above by $(h / n) \sum p_{i}$, which is of order $h \Delta / n$ in the most interesting case, in which $\sum p_{i}$ is roughly equal to $\Delta$. We thus need $h$ to be of order at least $n / \Delta$, giving a running time of order $(n / \Delta)^{2}$, while we are aiming for a sublinear running time of order $\tilde{O}(n / \Delta)$.

Our algorithm is based on a different idea by querying $p_{t}$ at a different precision each time, namely, by querying $p_{s_{t}}$ at precision $\epsilon_{t}=t / h$ on the $t$ th draw, and considering the estimator

$$
\frac{n}{h} \sum_{t=1}^{h} \mathbf{I}\left[p_{s_{t}} \geq \epsilon_{t}\right]
$$

for the sum $\sum_{i} p_{i}$. In expectation, this estimator is equal to $n$ times

$$
\frac{1}{h} \sum_{t=1}^{h} \operatorname{Pr}\left[p_{s_{t}} \geq \epsilon_{t}\right]=\frac{1}{h} \sum_{t=1}^{h} \operatorname{Pr}\left[p_{s_{t}} \geq \frac{t}{h}\right],
$$

with $s_{t}$ denoting an integer chosen uniformly at random from $[1: n]$. This is a Riemann sum approximation to the well-known expression

$$
\mathbf{E}\left[p_{s}\right]=\int_{0}^{1} d x \operatorname{Pr}\left[p_{s} \geq x\right],
$$

and it differs from this integral by an error $O\left(\frac{1}{h}\right)$. In the most interesting case, in which $\sum_{i} p_{i}$ is of order $\Delta$, concentration again requires $h$ to be of order at least $n / \Delta$, which also guarantees that the error $O(1 / h)$ from the Riemann sum approximation does not dominate the expectation $\mathbf{E}\left[p_{s}\right]=\frac{1}{n} \sum_{i} p_{i}$. But now we query $p_{s}$ at the highest resolution $\epsilon_{1}=1 / h$ only once, leading to a much faster running time. In fact, up to log factors, the running time will be dominated by the first few queries, giving a running time of $\tilde{O}(h)=\tilde{O}(n / \Delta)$, as desired.

In the next section, we proceed with the algorithm's formal description and analysis.

\section{I. A Multiscale Algorithm for Approximating Vector Sum}

Algorithm 1, MultiScaleVectorSum, replaces the standard sampling to estimate the sum $\sum_{i} p_{i}$ by a multiscale version that spends only a small amount of time at the computationally intensive scales requiring high precision. In addition to the 
black-box oracle vectorAccess $(\cdot)$, this algorithm takes three other parameters: $\Delta \in(1, n)$ and $c>1$ as defined in VECTORSum, and a confidence parameter $\delta \in(0,1)$. This algorithm uses randomization, and we will show that it correctly solves VeCTORSUm with probability at least $1-\delta$.

Our algorithm implements the strategy discussed above except for one modification: instead of sampling at a different precision $\epsilon_{t}$ each time, we sample at each precision a constant number of times $\tau$, where $\tau$ depends on the desired success probability, given a total number of queries equal to $L=\tau h$, where $h=\Theta(n / \Delta)$ with the implicit constant in the $\Theta$-symbol depending on $c$ in such a way that it grows with $(c-1)^{-2}$ as $c \rightarrow 1 .^{2}$

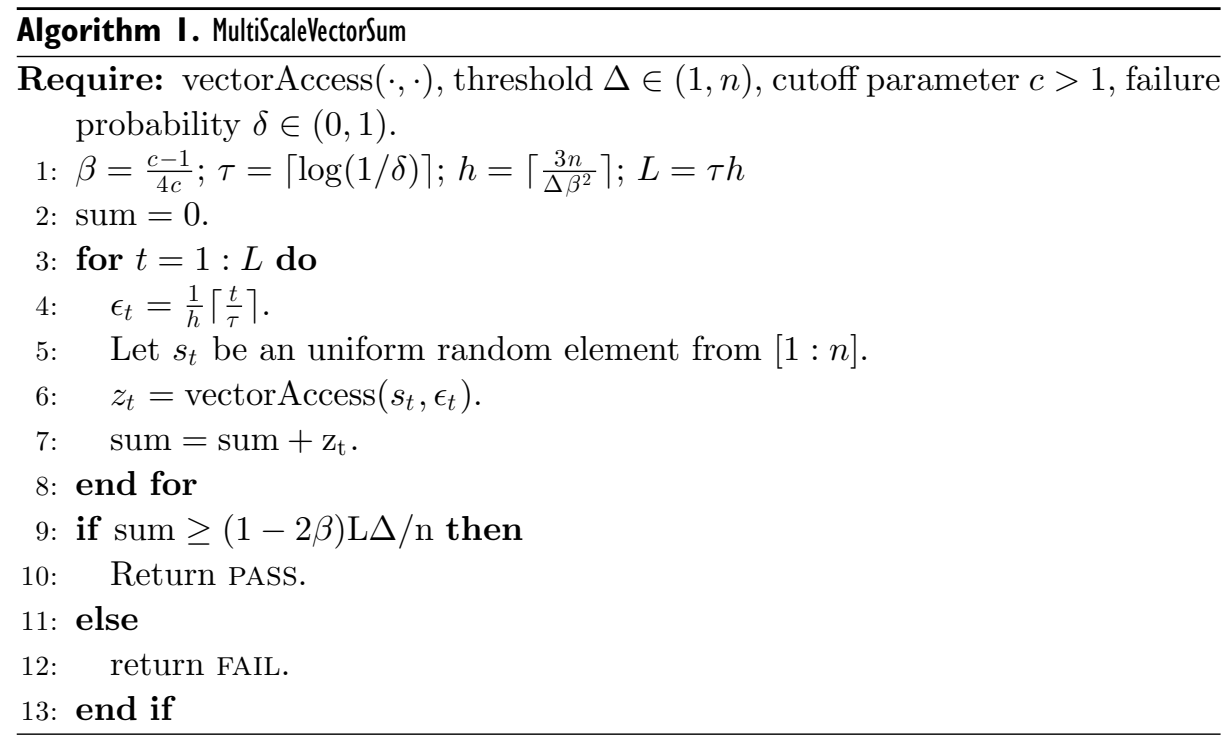

Theorem 3.2. (Multiscale Vector Sum.) For every $\mathbf{p} \in(0,1)^{n}$ accessible by vectorAccess $(\cdot)$, threshold $\Delta \in(1, n)$, robust parameter $c>1$, and failure parameter $\delta \in(0,1)$, the method MultiScaleVectorSum (vectorAccess $(\cdot), \Delta, c, \delta)$ correctly solves VECTORSUM with probability at least $(1-\delta)$ and costs

$$
O\left(\frac{n}{\Delta}\left(\frac{1}{c-1}\right)^{2} \log \left(\frac{n}{\Delta(c-1)}\right) \log \left(\frac{2}{\delta}\right)\right) .
$$

\footnotetext{
${ }^{2}$ Somewhat arbitrarily, but conveniently for our notation and proofs, we introduce the $c$ dependence of our constructions through the variable $\beta(c-1) / 4 c$; in terms of this variable, we write the lower cutoff $\Delta / c$ as $\Delta(1-4 \beta)$, and use the midpoint $\Delta(1-2 \beta)$ between $\Delta$ and $\Delta / c$ as the cutoff for the algorithm to decide between PASS and FAIL.
} 
Proof. By steps 3-7 of Algorithm 1, for every constant $c>1$, the cost of the algorithm is

$$
\sum_{t=1}^{L} \frac{1}{\epsilon_{t}}=\tau \sum_{i=1}^{h} \frac{h}{i} \leq L(1+\log h)=O\left(\frac{n}{\Delta}\left(\frac{1}{c-1}\right)^{2} \log \left(\frac{n}{\Delta(c-1)}\right) \log \left(\frac{2}{\delta}\right)\right) .
$$

We now prove the correctness of the algorithm.

After the initialization steps 1 and 2, Algorithm 1 computes the multiscale parameters $\epsilon_{t}$ and applies sampling to calculate the sum

$$
Q=\sum_{t=1}^{L} z_{t}=\sum_{t=1}^{L} \mathbf{I}\left[p_{s_{t}} \geq \epsilon_{t}\right]
$$

where $s_{1}, \ldots, s_{L}$ are chosen i.i.d. uniformly at random from $[1: n]$. The expectation of $Q$ is easily estimated in terms of the bounds

$$
\begin{aligned}
\mathbf{E}[Q] & =\frac{1}{n} \sum_{k=1}^{n} \sum_{t=1}^{L} \mathbf{I}\left[\epsilon_{t} \leq p_{k}\right]=\frac{1}{n} \sum_{k=1}^{n} \sum_{t=1}^{L} \mathbf{I}\left[\lceil t / \tau\rceil \leq h p_{k}\right] \\
& =\frac{\tau}{n} \sum_{k=1}^{n} \sum_{i=1}^{h} \mathbf{I}\left[i \leq h p_{k}\right]=\frac{\tau}{n} \sum_{k=1}^{n}\left\lfloor h p_{k}\right\rfloor \leq \frac{\tau}{n} \sum_{k=1}^{n} h p_{k}=\frac{L}{n} \sum_{k=1}^{n} p_{k}
\end{aligned}
$$

and

$$
\mathbf{E}[Q] \geq \frac{\tau}{n} \sum_{k=1}^{n}\left(h p_{k}-1\right)=\frac{L}{n} \sum_{k=1}^{n} p_{k}-\tau
$$

We thus use $\frac{n}{L} Q$ as an estimate of $\sum_{k=1}^{n} p_{k}$ when we decide whether to output PASS in step 9.

Assume first that $\sum p_{k} \geq \Delta$. Since

$$
\tau \leq \beta^{2} \frac{L \Delta}{3 n} \leq \beta \frac{L \Delta}{n},
$$

we then have

$$
\mathbf{E}[Q] \geq \frac{L \Delta}{n}-\tau \geq(1-\beta) \frac{L \Delta}{n},
$$

implying that

$$
(1-\beta) \mathbf{E}[Q] \geq(1-2 \beta) \frac{L \Delta}{n} .
$$


This allows us to use the multiplicative Chernoff bound in the form of Lemma 6.1 to conclude that

$$
\begin{aligned}
\operatorname{Pr}\left[Q \leq(1-2 \beta) \frac{L \Delta}{n}\right] \leq & \operatorname{Pr}[Q \leq(1-\beta) \mathbf{E}[Q]] \leq \exp \left(-\frac{\beta^{2}}{2} \mathbf{E}[Q]\right) \\
& \leq \exp \left(-\frac{3}{8} \frac{\beta^{2} L \Delta}{n}\right) \leq \delta,
\end{aligned}
$$

where we used $\beta \leq 1 / 4$ in the last step.

On the other hand, if $\sum p_{k} \leq \Delta / c=(1-4 \beta) \Delta$, we bound

$$
\mathbf{E}[Q] \leq \frac{L \Delta}{n}(1-4 \beta)
$$

which in turn implies that

$$
(1+2 \beta) \mathbf{E}(Q) \leq(1-2 \beta) \frac{L \Delta}{n} .
$$

Using the multiplicative Chernoff bound in the form of Lemma 6.1 (part 3), this gives

$$
\operatorname{Pr}\left[Q \geq(1-2 \beta) \frac{L \Delta}{n}\right] \leq \exp \left(-\beta^{2} \frac{L \Delta}{n} \frac{1-2 \beta}{1+2 \beta}\right) \leq \exp \left(-\frac{\beta^{2} L \Delta}{3 n}\right) \leq \delta,
$$

where we again used $\beta \leq 1 / 4$.

Thus, MultiScaleVectorSum (vectorAccess $(\cdot), \Delta, c, \delta)$ correctly solves VECTORSUM with probability at least $1-\delta$.

\section{Multiscale Matrix Sampling}

In this section, we consider SignificantMatrixColumns in a slightly more general matrix access model than what we defined in Sections 1 and 3. The extension of the model is also needed in our PageRank approximation algorithm, which we will present in the next section.

\section{I. Notation: Sparse Vectors}

To better specify this model and the subsequent algorithms, we first introduce the notation of sparse vector introduced in [Gilbert et al. 92] for MatwaB. Suppose $\mathbf{a}=\left(a_{1}, \ldots, a_{n}\right) \in \mathbb{R}^{n}$ is a vector. Let $\operatorname{nnz}(\mathbf{a})$ denote the number of nonzero elements in a. Let $\operatorname{Sparse}(\mathbf{a})$ denote the sparse form of vector a by "squeezing out" any zero elements in a. Conceptually, one can view $\operatorname{Sparse}(\mathbf{a})$ as a list of 
nnz(a) index-entry pairs, one for each nonzero element and its index in a. For example, we can view Sparse $([0,0.3,0.5,0,0.2])$ as $((2,0.3),(3,0.5),(5,0.2))$.

A sparse vector can be easily implemented using a binary search tree. ${ }^{3}$ Throughout this paper, we shall make use of the following simple proposition.

Proposition 4.I. For $\mathbf{a}, \mathbf{b} \in \mathbb{R}^{n}, \mathbf{a}+\mathbf{b}$ can be implemented in time $O(\operatorname{nnz}(\mathbf{b}) \cdot \log n)$ by saving the result in the data structure of $\mathbf{a}$.

Proof. Each sparse vector can be implemented as a balanced binary search tree, where the index of an entry serves as the entry's key. When performing the addition, we update the binary search tree of $\mathbf{a}$ by inserting the elements of $\mathbf{b}$ into it one by one (and updating existing entries whenever needed). By the standard theory of binary search trees, each such insertion operation takes $O(\log n)$ time.

In the rest of the paper, without further elaboration, we assume that all vectors are expressed in this sparse form. We also adopt the following notation: let Sparse([ ]) denote the all-zeros vector in the sparse form, and for $i \in[1: n]$ and $b \in \mathbb{R}-\{0\}$, let Sparse $(i, b)$ denote the sparse vector with only one nonzero element $b$ located in the $i$ th place in the vector. In addition, we will use the following notation: For two $n$-place vectors $\mathbf{a}=\left(a_{1}, \ldots, a_{n}\right)$ and $\mathbf{b}=\left(b_{1}, \ldots, b_{n}\right)$ and parameters $\epsilon \in \mathbb{R}$ and $C>0$, we use $\mathbf{a} \leq C \cdot \mathbf{b}+\epsilon$ to denote $a_{i} \leq C \dot{b}_{i}+\epsilon$, $\forall i \in[1: n]$.

\subsection{The Matrix Access Model}

In the model that we will consider in the rest of this section, we can access an unknown $n \times n$ right-stochastic matrix $M=\left(m_{i, j}\right)$ using queries of the form matrixAccess $(i, \epsilon, \lambda, p)$, where $i \in[1: n]$ specifies a row, $\epsilon \in(0,1]$ specifies a required additive precision, $\lambda \in(0,1]$ specifies a multiplicative precision, and $p \in(0,1]$ specifies the probability requirement. This query will return a sparse vector $\tilde{\mathbf{m}}_{i}=\operatorname{Sparse}\left(\left[\tilde{m}_{i, 1}, \ldots, \tilde{m}_{i, n}\right]\right)$ such that

- with probability at least $1-p$,

$$
(1-\lambda) \cdot \mathbf{m}_{i}-\epsilon \leq \tilde{\mathbf{m}}_{i} \leq(1+\lambda) \cdot \mathbf{m}_{i}+\epsilon,
$$

where $\mathbf{m}_{i}=M(i, \cdot)$ denotes the $i$ th row of matrix $M$, and

\footnotetext{
${ }^{3}$ For average-case rather than worst-case guarantees, a hash table is a typical implementation choice.
} 
- with probability at most $p$ (the query may fail), $\tilde{\mathbf{m}}_{i}$ can be any sparse vector.

We refer to this black-box model as the probabilistic sparse-and-approximate row-access model with additive/multiplicative errors. For constant integers

$$
c_{1}, c_{2}, c_{3}, c_{4}>0
$$

we say that matrixAccess is a $\left(c_{1}, c_{2}, c_{3}, c_{4}\right)$-SARA model if for all $i \in[1: n], \epsilon \in$ $(0,1), \lambda \in(0,1)$, and $p \in(0,1)$, the cost of calling $\tilde{\mathbf{m}}_{i}=\operatorname{matrix} \operatorname{Access}(i, \epsilon, \lambda, p)$ and that of calling $\operatorname{nnz}\left(\tilde{\mathbf{m}}_{i}\right)$ are bounded above by

$$
c_{1}\left(\frac{1}{\lambda}\right)^{c_{2}}\left(\frac{\log ^{c_{3}}(1 / \epsilon)}{\epsilon}\right)\left(\log ^{c_{4}} n\right) \log \left(\frac{1}{p}\right) .
$$

\subsection{The Matrix Problem}

In this section, we give a solution to the following abstract problem.

Problem 4.2. (SignificantMatrixColumns.) Given an $n \times n$ right-stochastic matrix $M$ in the $\left(c_{1}, c_{2}, c_{3}, c_{4}\right)$-SARA model, a threshold $\Delta$, and a positive constant $c>1$, return a sparse vector cSum with the property that for all $j \in[1: n]$, if $\operatorname{ColumnSum}(M, i) \geq \Delta$, then $\operatorname{cSum}(j) \neq 0$, and if $\operatorname{ColumnSum}(M, i)<\Delta / c$, then $\operatorname{cSum}(j)=0$.

\subsection{Understanding the Impact of Additive/Multiplicative Errors}

Our algorithm for SignificantMatrixColumns is straightforward. At a high level, it simultaneously applies Algorithm 1 to all columns of the unknown matrix. It uses a sparse-vector representation for efficient bookkeeping of the columns with large sum according to the sampled data. Our analysis of this algorithm is similar to that presented in Theorem 3.2 for VECTORSum, since we can use the union bound over the columns to reduce the analysis to a single column. The only technical difference is the handling of the additive/multiplicative errors.

To understand the impact of these errors, we consider a vector $\mathbf{p}=\left(p_{1}, \ldots, p_{n}\right)$ in $[0,1]^{n}$ and choose $\epsilon_{t}, t=1, \ldots, L$, as in Algorithm 1. Fix $\phi, \lambda \in(0,1 / 2)$, and suppose that we access $p_{i}$ with multiplicative error $\lambda$ and additive error $\phi \cdot \epsilon_{t}$. We will show that if this returns a number $\tilde{p}_{i} \geq \epsilon_{t}$, then the actual value of $p_{i}$ is at least $\rho \epsilon_{t}$, where $\rho=1-\lambda-\phi$. To see this, we bound

$$
p_{i} \geq(1+\lambda)^{-1}\left(\tilde{p}_{i}-\phi \cdot \epsilon_{t}\right) \geq(1+\lambda)^{-1}(1-\phi) \epsilon_{t} .
$$

Since $(1-\phi) /(1+\lambda) \geq(1-\lambda-\phi)$, this implies that $p_{i} \geq \rho \epsilon_{t}$, as desired. 
In a similar way, it is easy to see that $p_{i} \geq \rho^{-1} \epsilon_{t}$ implies that $\tilde{p}_{i} \geq \epsilon_{t}$. Indeed, if $p_{i} \geq \rho^{-1} \epsilon_{t}$, then

$$
\tilde{p}_{i} \geq(1-\lambda) p_{i}-\phi \cdot \epsilon_{t} \geq\left(\frac{1-\lambda}{1-\lambda-\phi}-\phi\right) \epsilon_{t} .
$$

The lower bound is clearly larger than $\epsilon_{t}$, showing that $\tilde{p}_{i} \geq \epsilon_{t}$.

For $s_{1} \ldots, s_{L} \in[1: n]$, the sum

$$
\tilde{Q}=\sum_{t=1}^{L} \mathbf{I}\left[\tilde{p}_{s_{t}} \geq \epsilon_{t}\right]
$$

can therefore be bounded from below and above by

$$
Q_{-}=\sum_{t=1}^{L} \mathbf{I}\left[p_{s_{t}} \geq \rho^{-1} \epsilon_{t}\right] \quad \text { and } \quad Q_{+}=\sum_{t=1}^{L} \mathbf{I}\left[p_{s_{t}} \geq \rho \epsilon_{t}\right]
$$

respectively:

$$
Q_{-} \leq \tilde{Q} \leq Q_{+}
$$

Finally, we also note that if we access $p_{i}$ with multiplicative error $\lambda$ and additive error $\phi \cdot \epsilon_{t}$, then this returns a number that is never larger than $\rho^{-1}$. Indeed, this follows by bounding $\tilde{p}_{i}$ by $1+\lambda+\phi \cdot \epsilon_{t} \leq 1+\lambda+\phi \leq \rho^{-1}$.

\subsection{A Multiscale Algorithm}

In this section, we present the multiscale algorithm in full detail and proceed with an analysis of its running time and correctness. The algorithm is essentially an extension of Algorithm 1, applying the VECTORSUM algorithm to all columns in parallel. Since the call to VECTORACESSS has now been replaced by a combined additive-multiplicative method, the constant $\beta$ is set to a slightly smaller value than in Algorithm 1. In addition to the constants $\beta, \tau, h, L$ that are used in Algorithm 1, we also have the constant $\lambda$ for the value of multiplicative approximation needed and $\phi$ for the additive approximation needed. Lastly, $p$ is the desired success probability of the row approximation procedure (matrixAccess) invoked throughout the algorithm. We note that these constants are defined to allow complete and rigorous analysis of our algorithm and its correctness. Since the multiscale algorithm will essentially be implementing Algorithm 1 over all columns, we will need a method that can return all elements in a row that fall within a certain bin; we call it the rangeIndicator method: for a sparse vector a and $l, u \in \mathbb{R}$ such that $l<u$, we have that $\mathbf{b}=\operatorname{rangeIndicator}(\mathbf{a}, l, u)$ returns a 
sparse vector $\mathbf{b}$ such that for all $i \in[1: n]$,

$$
\mathbf{b}(i)= \begin{cases}1 & \text { if } l \leq \mathbf{a}(i) \leq u, \\ 0 & \text { otherwise }\end{cases}
$$

For example, rangeIndicator(Sparse $([0,0.3,0.5,0,0.2]), 0.1,0.3)$ returns the sparse form of $[0,1,0,0,1]$. We shall use the following simple proposition.

Proposition 4.3. rangeIndicator $(\mathbf{a}, l, u)$ takes $O(\operatorname{nnz}(\mathbf{a}) \log n)$ time.

Proof. The sparse vector nnz(a) is implemented using a binary search tree; one can therefore scan its contents using, say, an in-order scan and insert each element in the range $[l, u]$ into a sparse vector $\mathbf{b}$, initially empty. The in-order scan costs $O(\operatorname{nnz}(\mathbf{a}))$ time, and each insertion into $b \operatorname{costs} O(\log n)$ time, giving the desired result.

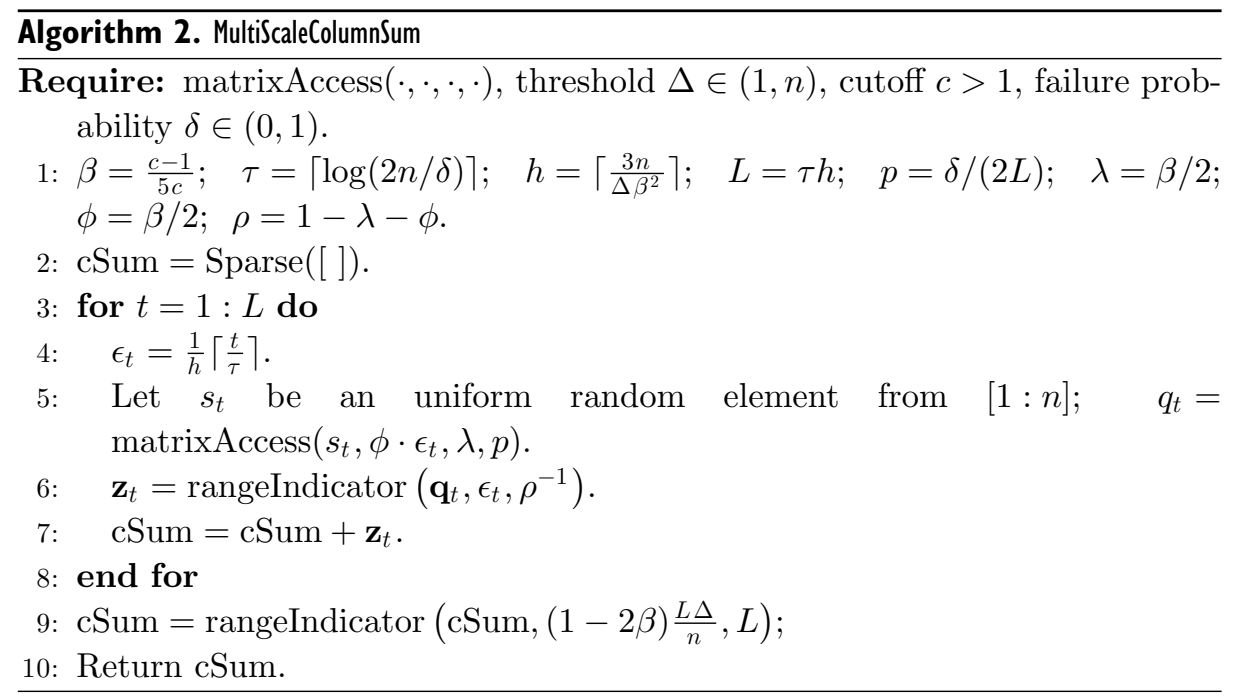

We are now ready to state our main theorem.

Theorem 4.4. (Multiscale column sum.) For every right-stochastic matrix $M$ accessible by matrixAccess, threshold $\Delta \in(1, n)$, robust parameter $c>1$, and failure parameter $\delta \in(0,1)$, with probability at least $(1-\delta)$,

$$
\operatorname{cSum}=\text { MultiScaleVectorSum }(\operatorname{vectorAccess}(\cdot), \Delta, c, \delta)
$$

correctly solves SignifiCAntMatRixColumns. 
Furthermore, if matrixAccess is a $\left(c_{1}, c_{2}, c_{3}, c_{4}\right)$-SARA model, then the cost of MultiScaleVectorSum (vectorAccess $(\cdot), \Delta, c, \delta)$ is

$$
O\left(c_{1}\left(\frac{n}{\Delta}\right)\left(\frac{1}{c-1}\right)^{c_{2}+3} \log ^{c_{3}+2}\left(\frac{1}{c-1}\right) \log ^{c_{3}+c_{4}+3} n \log ^{2}\left(\frac{2}{\delta}\right)\right) .
$$

Proof. The cost of the algorithm is dominated by the sparse matrix operations in lines 5-7 of Algorithm 2, plus the cost of the last operation in line 9. Using our access model together with Propositions 4.1 and 4.3, we see that the cost of the steps in lines $5-7$ at time $t$ is of order

$$
\begin{aligned}
& O\left(c_{1}\left(\frac{1}{\beta}\right)^{c_{2}} \frac{\log ^{c_{3}}\left(\frac{1}{\beta \epsilon_{t}}\right)}{\beta \epsilon_{t}} \log ^{c_{4}+1} n \log \left(\frac{2 L}{\delta}\right)\right) \\
& \quad \leq O\left(c_{1} \frac{h}{\lceil t / \tau\rceil} \log ^{c_{3}} h\left(\frac{1}{\beta}\right)^{c_{2}+1} \log ^{c_{4}+1} n \log \left(\frac{2 n}{\Delta \beta \delta}\right)\right) .
\end{aligned}
$$

Note that this includes the extra factor of $\log n$ from Proposition 4.1, a factor that is absent in the sparseness of $\mathbf{q}_{t}$ and $\mathbf{z}_{t}$.

Summing over $t$ gives a running time of order

$$
\begin{aligned}
& O\left(c_{1} L \log ^{c_{3}+1} h\left(\frac{1}{\beta}\right)^{c_{2}+1} \log ^{c_{4}+1} n \log \left(\frac{2 n}{\Delta \beta \delta}\right)\right) \\
& =O\left(c_{1}\left(\frac{n}{\Delta}\right) \log ^{c_{3}+1}\left(\frac{n}{\Delta \beta}\right)\left(\frac{1}{\beta}\right)^{c_{2}+3} \log ^{c_{4}+1} n \log \left(\frac{2 n}{\Delta \beta \delta}\right) \log \left(\frac{2}{\delta}\right)\right) \\
& =O\left(c_{1}\left(\frac{n}{\Delta}\right)\left(\frac{1}{\beta}\right)^{c_{2}+3} \log ^{c_{3}+2}\left(\frac{1}{\beta}\right) \log ^{c_{3}+c_{4}+3} n \log ^{2}\left(\frac{2}{\delta}\right)\right) .
\end{aligned}
$$

To estimate the cost of the last step of the algorithm, we bound the sparseness of cSum at the completion of the FOR loop by nnz(cSum $) \leq \sum_{t} n n z\left(\mathbf{z}_{t}\right)$ and then apply Proposition 4.3 once more, giving a cost that is of the same order as the total cost of the algorithm accrued up to this step.

To prove the correctness of the algorithm, we first note that with probability at least $(1-p)^{L} \geq 1-p L$, each of the $L$ calls of matrixAccess in line 5 will return a sparse vector satisfying the bound (4.1). Next, we apply the union bound to reduce the focus of the analysis to a single column:

$\operatorname{Pr}\{$ MultiScaleColumnSum is unsuccessful $\}$

$\leq \sum_{i=1}^{n} \operatorname{Pr}\{$ MultiScaleColumnSum is unsuccessful on column $i\}$. 
When considering column $i$, we now let $\mathbf{p}=\left(p_{1}, \ldots, p_{n}\right)^{T}=M(\cdot, i)$, the $i$ th column of $M$. In other words, $p_{j}=m_{i, j}$ for all $j \in[1: n]$. Note that the $i$ th entry of cSum after step 8 is of the form (4.2). With the bound (4.4) taken into account, our proof will be very similar to that of Theorem 3.2.

We first consider the case that $\sum_{i} p_{i} \geq \Delta$, in which case we bound

$$
\mathbf{E}\left[Q_{-}\right] \geq \frac{L \rho}{n} \sum_{k} p_{k}-\tau \geq \frac{\Delta L}{n}\left[\rho-\frac{\beta^{2}}{3}\right]=\frac{\Delta L}{n}\left[1-\beta-\frac{\beta^{2}}{3}\right] .
$$

Multiplying both sides by $(1-\beta)$, we obtain

$$
(1-\beta) \mathbf{E}\left[Q_{-}\right] \geq \frac{\Delta L}{n}(1-2 \beta) .
$$

Combined with the bound (4.4) and the multiplicative Chernoff bound (Lemma 6.1), this shows that conditioned on matrixAccess returning a sparse vector satisfying the bound (4.1) in each instance in line 5 , we get

$$
\operatorname{Pr}\left\{\mathrm{cSum}(i) \leq(1-2 \beta) \frac{\Delta L}{n}\right\} \leq \exp \left(-\frac{\beta^{2}}{2} \mathbf{E}\left[Q_{-}\right]\right) \leq \exp \left(-\frac{3}{8} \frac{\beta^{2} L \Delta}{n}\right) \leq \frac{\delta}{2 n} \text {. }
$$

In a similar way, if $\sum_{k} p_{k} \leq \Delta / c=\Delta(1-5 \beta)$, we bound

$$
\mathbf{E}\left[Q_{+}\right] \leq \frac{\Delta L}{n \rho}(1-5 \beta)=\frac{\Delta L}{n} \frac{1-5 \beta}{1-\beta},
$$

implying that

$$
(1+2 \beta) \mathbf{E}\left[Q_{+}\right] \leq \frac{\Delta L}{n}(1-2 \beta)
$$

and hence

$$
\begin{aligned}
\operatorname{Pr}\left\{\operatorname{cSum}(i) \geq(1-2 \beta) \frac{\Delta L}{n}\right\} & \leq \exp \left(-\beta^{2} \frac{\Delta L}{n} \frac{1-2 \beta}{1+2 \beta}\right) \\
& \leq \exp \left(-\frac{\Delta L \beta^{2}}{3 n}\right) \leq \frac{\delta}{2 n}
\end{aligned}
$$

again conditioned on matrixAccess returning a sparse vector satisfying the bound (4.1) in each instance in line 5.

Thus the total failure probability is at most $p \cdot L+n \delta / 2 n=\delta$, as desired.

\section{Identifying Nodes with Significant PageRank}

\section{I. Robust Approximation of Personalized PageRanks}

We now present our main subroutine for SignificantPageRAnks, which, we recall, addresses the following problem: Given a directed graph $G=(V, E)$, a 
threshold value $1 \leq \Delta \leq|V|$, and a positive constant $c>1$, compute a subset $S \subseteq V$ with the property that $S$ contains all vertices of PageRank at least $\Delta$ and no vertex with PageRank less than $\Delta / c$.

Let PPR denote the personalized PageRank matrix of $G$ defined in the introduction, where we recall that $\operatorname{PPR}(i, j)$ is equal to the personalized PageRank contribution of node $i$ to node $j$ in $G$. Under this notation, the SignificantPAGERANKS problem can be viewed as a SignificAntMatrixColumns problem if we can develop an efficient procedure for accessing the rows of PPR. This procedure, which we refer to as PPRmatrixAccess $(\cdot)$, takes a row number $i$, an additive precision parameter $\epsilon$, a multiplicative precision parameter $\lambda$, and success probability $p$, and returns a sparse vector $\tilde{\mathbf{m}}_{i}=\operatorname{Sparse}\left(\left[\tilde{m}_{i, 1}, \ldots, \tilde{m}_{i, n}\right]\right)$ such that

- with probability at least $1-p$,

$$
(1-\lambda) \cdot \mathbf{m}_{i}-\epsilon \leq \tilde{\mathbf{m}}_{i} \leq(1+\lambda) \cdot \mathbf{m}_{i}+\epsilon
$$

where $\mathbf{m}_{i}=\operatorname{PPR}(i, \cdot)$, and

- with probability at most $p, \tilde{\mathbf{m}}_{i}$ can be any sparse vector.

Our algorithm for PPRmatrixAccess $(\cdot)$ uses the following key observation that connects personalized PageRank with the hitting probability of a Markov model.

Remark 5.I. $\operatorname{PPR}(v, j)$ is equal to the success probability that a random walk starting at $v$ and independently terminating at each time step with probability $\alpha$ hits $j$ just before termination.

Proof. Let $\mathbf{1}_{v}$ be the indicator vector of $v$. Solving the system given by

PersonalizedPageRank $(v, \cdot)=\alpha \mathbf{1}_{v}+(1-\alpha) \operatorname{PersonalizedPageRank}(v, \cdot) D^{-1} A$, one obtains

$$
\begin{aligned}
\operatorname{PersonalizedPageRank}(v, \cdot) & =\alpha \mathbf{1}_{v}\left(I-(1-\alpha) D^{-1} A\right)^{-1} \\
& =\alpha \mathbf{1}_{v} \sum_{i=0}^{\infty}\left((1-\alpha) D^{-1} A\right)^{i} .
\end{aligned}
$$

The observation then follows directly from the last equation.

Our Algorithm 3 for PPRmatrixAccess conducts a careful simulation of such restarting random walks. As such, it needs only an oracle access to a random out-link of a given node. 


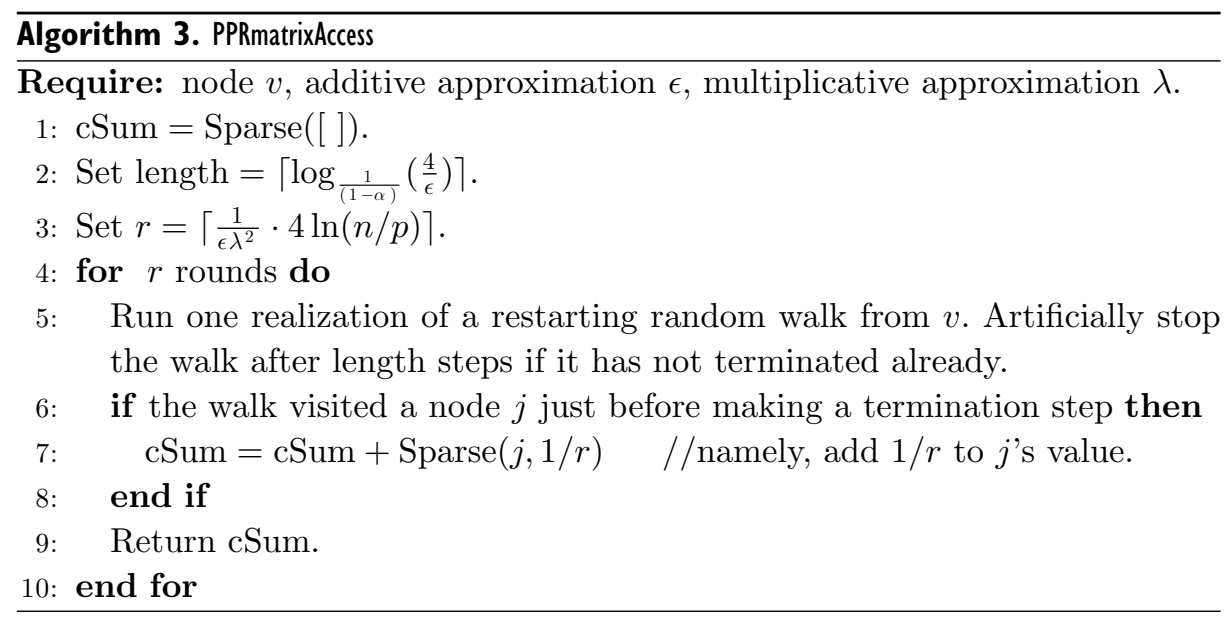

Theorem 5.2. For every node $v$, values $0<\epsilon<1,0<\lambda<1,0<\alpha<1$, and success probability $0<p<1$, PPRmatrix Access $(v, \epsilon, \lambda, p)$ is a

$$
\left(10 \max \left\{\log ^{-1}\left(\frac{1}{1-\alpha}\right), 1\right\}, 2,1,2\right) \text {-SARA model. }
$$

In particular, its running time is bounded above by

$$
O\left(\frac{\ln ^{2}(n) \ln (1 / p) \log \left(\epsilon^{-1}\right)}{\epsilon \lambda^{2}}\right) .
$$

Proof. We begin by analyzing the running time guarantee. The algorithm performs $\left\lceil\frac{1}{\epsilon \lambda^{2}} \cdot 4 \ln (n / p)\right\rceil$ rounds, where at each round, it simulates a random walk with termination probability of $\alpha$ for at most length steps. Each step is simulated by taking a uniform sample ("termination" step) with probability $\alpha$ and by choosing a random out-link with probability $1-\alpha$. The update of cSum in line 7 takes at most $\log n$ (see Proposition 4.1). Thus the total number of queries used is

$$
\begin{gathered}
\left\lceil\frac{4 \ln (n / p)}{\epsilon \lambda^{2}}\right\rceil \cdot\left\lceil\log _{\frac{1}{(1-\alpha)}}\left(\frac{4}{\epsilon}\right)\right\rceil \log (n) \leq\left\lceil\frac{4 \ln (n / p)}{\epsilon \lambda^{2}}\right\rceil \cdot\left\lceil\frac{\log \left(\frac{4}{\epsilon}\right)}{\log \left(\frac{1}{1-\alpha}\right)}\right\rceil \log (n) \\
\leq(8+2) \max \left\{\log ^{-1}\left(\frac{1}{1-\alpha}\right), 1\right\} \frac{\ln ^{2}(n) \log (1 / p) \log \left(\epsilon^{-1}\right)}{\epsilon \lambda^{2}} .
\end{gathered}
$$

We now prove the guarantees on the returned vector cSum (line 9 in the algorithm). Given a node $j$, denote by $p_{k}(v, j)$ the contribution to $j$ from restarting 
walks originating at $v$ that are of length at most $k$, namely,

$$
\left.p_{k}(v, j)=\alpha \mathbf{1}_{v} \sum_{i=0}^{k}(1-\alpha) D^{-1} A\right)^{i} .
$$

We ask how much is contributed to $j$ 's entry from restarting walks of length greater than or equal to $k$. The contribution is at most $(1-\alpha)^{k}$, since the walk needs to survive at least $k$ consecutive steps. Taking $(1-\alpha)^{k} \leq \epsilon / 4$ will guarantee that at most $\epsilon / 4$ is lost by considering only walks of length smaller than $k$, namely,

$$
\operatorname{PPR}(v, j)-\frac{\epsilon}{4} \leq p_{k}(v, j) \leq \operatorname{PPR}(v, j) .
$$

For this to hold, it suffices to take $k=\left\lceil\log _{\frac{1}{(1-\alpha)}}\left(\frac{4}{\epsilon}\right)\right\rceil$, the value to which the parameter length is set in step 2 .

Next, the algorithm computes an estimate of $p_{k}(v, j)$ by realizing walks of length at most $k$. This is the value of cSum at index $j$ returned by the algorithm. Denote this by $\hat{p}_{k}(v, j)$. The algorithm computes such an estimate (in line 7 ) by taking the average number of hits over $r$ trials (adding $1 / r$ per hit).

Now, if $\operatorname{PPR}(v, j) \geq \epsilon / 2$, then $p_{k}(v, j) \geq \epsilon / 4$, and by the multiplicative Chernoff bound (Lemma 6.1),

$$
\operatorname{Pr}\left(\hat{p}_{k}(v, j)>(1+\lambda) p_{k}(v, j)\right) \leq \exp (-\ln (n / p))
$$

and

$$
\operatorname{Pr}\left(\hat{p}_{k}(v, j)<(1-\lambda) p_{k}(v, j)\right) \leq \exp (-\ln (n / p)) .
$$

By the union bound, we can conclude that with probability $1-2 p / n$,

$$
(1-\lambda)\left(\operatorname{PPR}(v, j)-\frac{\epsilon}{4}\right) \leq \hat{p}_{k}(v, j) \leq(1+\lambda) \operatorname{PPR}(v, j)
$$

Similarly, if $\operatorname{PPR}(v, j)<\frac{\epsilon}{2}$, then $p_{k}(v, j)<\frac{\epsilon}{2}$, and by the multiplicative Chernoff bound (Lemma 6.1, part 3),

$$
\operatorname{Pr}\left(\hat{p}_{k}(v, j)>(1+\lambda) \frac{\epsilon}{2}\right) \leq \exp (-\ln (n / p))=\frac{p}{n} .
$$

Since $\lambda<1$, we therefore have $0 \leq \hat{p}_{k}(v, j) \leq \epsilon$ with probability at least $1-$ $p / n$. And since $\operatorname{PPR}(v, j)<\epsilon / 2$, we clearly have, with probability $1-p / n$,

$$
(1-\lambda) \operatorname{PPR}(v, j)-\epsilon \leq \hat{p}_{k}(v, j) \leq(1+\lambda) \operatorname{PPR}(v, j)+\epsilon,
$$

as required.

By the union bound, the complete claim holds with probability at least $1-p$. 


\subsection{A Tight Lower Bound for Solving the SignificantPageRanks Problem}

In this subsection, we present a corresponding lower bound for identifying all nodes with significant PageRank values. Our lower bound holds under the stringent model whereby one can access any node of interest in the graph in one unit of cost and the PageRank of the node accessed is given for free. We call such a model the strong query model. We first give a lower bound to illustrate the challenge for identifying nodes with significant PageRanks, even in graphs with only one significant node.

We then show that for every integral threshold $\Delta$ and precision $c$, there are instances whereby the output size of SignifiCANTPAgERAnKs is $\Omega(n / \Delta)$. Clearly, this also serves as a lower bound for the running time of any algorithm that solves the SignificantPageRAnks problem, regardless of the computational model used to compute the required output. We note that the running time of our algorithmic solution to SignificantPageRAnks is at most only a small polylogarithmic factor away from this bound.

For clarity of exposition, we present our lower bounds for $\alpha=0.5$. Similar lower bounds hold for every fixed $0<\alpha<1$.

Theorem 5.3. (Difficulty in identifying one significant node.) Let $\alpha=0.5$. For $n$ large enough, every algorithm making fewer than $n / 6 \Delta$ queries in the strong query model on graphs on $n$ nodes and threshold $\Delta \leq n / 9$ will fail with probability at least $1 / e$ to find a node with PageRank at least $\Delta$, on at least one graph on $n$ nodes.

Proof. The proof will apply Yao's minimax principle for analyzing randomized algorithms [Yao 77], which uses the average-case complexity of the deterministic algorithms to derive a lower bound on the randomized algorithms for solving a problem.

Given positive integers $n$ and $\Delta \leq n / 9$, we construct a family $\mathcal{F}$ of undirected graphs on $n$ nodes by taking a cycle subgraph on $n-d-1$ nodes and an isolated star subgraph on the remaining $d+1$ nodes, where we set $d=3 \Delta-1$. To complete the construction, we take a random labeling of the nodes. See Figure 1 for an illustration.

Let $A$ be a deterministic algorithm for the problem. We shall analyze the behavior of $A$ on a uniformly random graph from $\mathcal{F}$.

First, by solving the PageRank equation system, it is easy to check that each node on the cycle subgraph has PageRank value of 1 , the hub of the subgraph has PageRank $\frac{d}{3}+\frac{2}{3}$, and a leaf of the star subgraph has PageRank $\frac{2}{3}+\frac{1}{3 d}$. The only node with PageRank at least $\Delta$ is the hub of the star subgraph. 

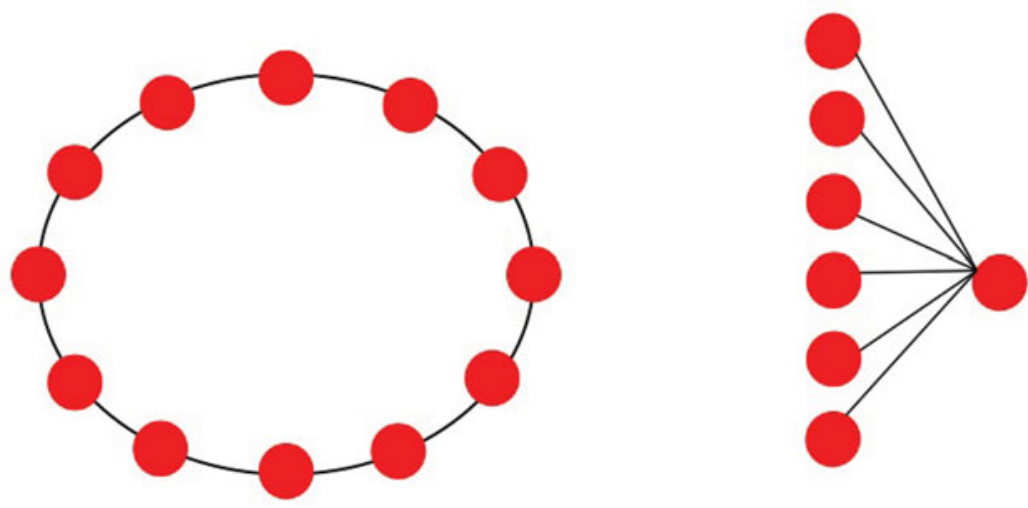

Figure I. An example illustrating the "cycle \& star" lower bound construction for PageRank computations.

Let $T$ be the number of queries the algorithm makes. The probability that none of the nodes of the star subgraph are found after $T$ queries by $A$ is at least

$$
\left(1-\frac{d+1}{n}\right)^{T} \geq \exp \left(-2 T \frac{d+1}{n}\right) \geq \exp (-1), \quad \text { for } T \leq \frac{n}{6 \Delta}=\frac{n}{2(d+1)}
$$

Here we used the fact that $1-x \geq \exp (-2 x)$ for $0 \leq x \leq 1 / 3$.

We define the cost of the algorithm as 0 if it has found a node of PageRank at least $\Delta$, and as 1 otherwise. Note that the cost of an algorithm equals its probability of failure. Then by Yao's minimax principle, every randomized algorithm that makes at most $n / 6 \Delta$ queries will have an expected cost of at least $1 / e$, i.e., a failure probability of at least $1 / e$ on at least one of the inputs.

Theorem 5.4. (Graphs with many significant nodes.) Let $\alpha=0.5$, let $\Delta$ be integral, and let $c$ be given. Then there are infinitely many $n$ such that there exists a graph on $n$ nodes whose output to SignificantPageRANks has size $\Omega(n / \Delta)$.

Proof. The construction is a variant of that used in the proof of Theorem 5.4. The graph comprises $n /(3 \Delta+1)$ identical copies of an undirected star graph on $d+1=3 \Delta$ nodes. An easy calculation with the PageRank equations shows that each hub has PageRank $\Delta+\frac{1}{3}$, and each leaf has PageRank

$$
\frac{2}{3}+\frac{1}{9 \Delta-3} \leq 1
$$


The number of nodes with PageRank at least $\Delta$ is therefore

$$
\frac{n}{d}=\Omega\left(\frac{n}{\Delta}\right)
$$

\section{Appendix: Concentration Bounds}

Lemma 6.I. (Multiplicative Chernoff bound.) Let $X=\sum_{i=1}^{n} X_{i}$ be a sum of independent (but not necessarily identical) Bernoulli random variables. Then the following hold:

1. For $0<\lambda<1$,

$$
\begin{aligned}
& \operatorname{Pr}[X<(1-\lambda) E[X]]<\exp \left(-\frac{\lambda^{2}}{2} E[X]\right), \\
& \operatorname{Pr}[X>(1+\lambda) E[X]]<\exp \left(-\frac{\lambda^{2}}{4} E[X]\right) .
\end{aligned}
$$

2. For $\lambda \geq 1$,

$$
\operatorname{Pr}[X>(1+\lambda) E[X]]<\exp \left(-\frac{\lambda E[X]}{3}\right)
$$

3. For every constant $\Delta \geq(1+\lambda) E[X]$,

$$
\operatorname{Pr}[X>\Delta]< \begin{cases}\exp \left(-\frac{\lambda^{2}}{4} \cdot \frac{\Delta}{1+\lambda}\right) & \text { if } 0<\lambda<1 \\ \exp \left(-\frac{\lambda}{3} \cdot \frac{\Delta}{(1+\lambda)}\right) & \text { if } \lambda \geq 1\end{cases}
$$

Proof. The case $0<\lambda<1$ is standard, and a proof can be found, for example, in [Motwani and Raghavan 95, Chapter 4]. For every $\lambda$, it is also shown therein that

$$
\operatorname{Pr}[X>(1+\lambda) \mu n] \leq\left(\frac{e^{\lambda}}{(1+\lambda)^{(1+\lambda)}}\right)^{\mu n}
$$

Now for $\lambda \geq 1$,

$$
\frac{e^{\lambda}}{(1+\lambda)^{(1+\lambda)}}<\exp \left(-\frac{\lambda^{2}}{2+\lambda}\right) \leq \exp \left(-\frac{\lambda}{3}\right),
$$

and the second item follows. 
We now prove the last item. Assume that

$$
\frac{\Delta}{(1+\lambda)}-E[X]>0
$$

(otherwise, the proof follows immediately from part 1). Define

$$
k=\left\lceil\frac{\Delta}{(1+\lambda)}-E[X]\right\rceil \text { and } Y=\sum_{i=1}^{n+k} Y_{i}
$$

where for $1 \leq i \leq n, Y_{i}=X_{i}$, and $n<i \leq n+k$, the $Y_{i}$ are independently distributed Bernoulli random variables each with expectation

$$
\frac{\left(\frac{\Delta}{(1+\lambda)}-E[X]\right)}{k} .
$$

Note that $k \geq 1$; the $Y_{i}$ are indeed Bernoulli random variables, since

$$
0<\frac{\left(\frac{\Delta}{1+\lambda}-E[X]\right)}{k} \leq 1
$$

and

$$
E[Y]=E[X]+\left(\frac{\Delta}{(1+\lambda)}-E[X]\right)=\frac{\Delta}{(1+\lambda)}
$$

Now,

$$
\begin{aligned}
& \operatorname{Pr}(X>\Delta)=\operatorname{Pr}\left(X>\frac{(1+\lambda) \Delta}{1+\lambda}\right) \\
& \quad \leq \operatorname{Pr}\left(Y>\frac{(1+\lambda) \Delta}{1+\lambda}\right)< \begin{cases}\exp \left(-\frac{\lambda^{2}}{4} \cdot \frac{\Delta}{1+\lambda}\right) & \text { if } \lambda<1, \\
\exp \left(-\frac{\lambda}{3} \cdot \frac{\Delta}{(1+\lambda)}\right) & \text { if } \lambda \geq 1 .\end{cases}
\end{aligned}
$$

The next-to-last inequality follows from the fact that $Y$ first-order stochastically dominates $X$, and the last inequality follows from parts 1 and 2 of the lemma.

Acknowledgments. We would like to thank the anonymous reviewers of Internet Mathemat$i c s$ and $W A W 2012$ for their valuable feedback and comments (an extended abstract of this paper appeared in $W A W$ 2012, pp. 41-53, under the title "A Sublinear Time Algorithm for PageRank Computations"). We thank Yevgeniy Vorobeychik, Elchanan Mossel, and Brendan Lucier for their suggestions at early stages of this work.

Funding. The research of the last-named author was supported in part by NSF grants CCF-1111270 and CCF-0964481. 


\section{References}

[Andersen et al. 06] Reid Andersen, Fan R. K. Chung, and Kevin J. Lang. "Local Graph Partitioning Using PageRrank Vectors." In FOCS, pp. 475-486, 2006.

[Andersen et al. 08] Reid Andersen, Christian Borgs, Jennifer T. Chayes, John E. Hopcroft, Vahab S. Mirrokni, and Shang-Hua Teng. "Local Computation of PageRank Contributions." Internet Mathematics 5 (2008), 23-45.

[Avrachenkov 07] K. Avrachenkov, N. Litvak, D. Nemirovsky, and N. Osipova. "Monte Carlo Methods in PageRank Computation: When One Iteration Is Sufficient." SIAM Journal on Numerical Analysis 45 (2007), 890-904.

[Bahmani et al. 10] Bahman Bahmani, Abdur Chowdhury, and Ashish Goel. "Fast Incremental and Personalized PageRank." PVLDB 4 (2010), 173-184.

[Berkhin 05] Pavel Berkhin. "Survey: A Survey on PageRank Computing." Internet Mathematics 2 (2005), 1-120.

[Brin and Page 98] Sergey Brin and Lawrence Page. "The Anatomy of a Large-Scale Hypertextual Web Search Engine." Computer Networks 30 (1998), 107-117.

[Cormode and Muthukrishnan 05] Graham Cormode and S. Muthukrishnan. "An Improved Data Stream Summary: The Count-Min Sketch and Its Applications." $J$. Algorithms 55 (2005), 58-75.

[Gilbert et al. 92] John R. Gilbert, Cleve Moler, and Robert Schreiber. "Sparse Matrices in Matlab: Design and Implementation." SIAM J. Matrix Anal. Appl. 13 (1992), 333-356.

[Goldreich 10] Oded Goldreich. "Introduction to Testing Graph Properties." In Property Testing, vol. 6390, edited by O. Goldreich, pp. 105-141. Berlin: Springer, 2010.

[Haveliwala 03] T. H. Haveliwala. "Topic-Sensitive PageRank: A Context-Sensitive Ranking Algorithm for Web Search." In Trans. Knowl. Data Eng 15 (2003) 784796.

[Jeh and Widom 03] Glen Jeh and Jennifer Widom. "Scaling Personalized Web Search." In $W W W$, pp. 271-279, 2003.

[Kannan 10] Ravindran Kannan. "Spectral Methods for Matrices and Tensors." In STOC, pp. 1-12, 2010.

[Kannan and Vempala 09] Ravi Kannan and Santosh Vempala. "Spectral Algorithms." Foundations and Trends in Theoretical Computer Science 4 (2009), 157-288.

[Motwani and Raghavan 95] Rajeev Motwani and Prabhaker Raghavan. Randomized Algorithms. Cambridge University Press, 1995.

[Page et al. 98] Lawrence Page, Sergey Brin, Rajeev Motwani, and Terry Winograd. "The PageRank Citation Ranking: Bringing Order to the Web." Technical report, Stanford University, 1998.

[Pandurangan et al. 06] Gopal Pandurangan, Prabhakar Raghavan, and Eli Upfal. "Using PageRank to Characterize Web Structure." Internet Mathematics 3 (2006), 1-20.

[Rubinfeld and Shapira 11] Ronitt Rubinfeld and Asaf Shapira. "Sublinear Time Algorithms." SIAM Journal on Discrete Math 25 (2011), 1562-1588. 
[Spielman and Teng 13] Daniel Spielman and Shang-Hua Teng. "A Local Clustering Algorithm for Massive Graphs and Its Application to Nearly Linear Time Graph Partitioning." SIAM J. Comput. 42 (2013), 1-26.

[Yao 77] Andrew Chi-Chih Yao. "Probabilistic Computations: Toward a Unified Measure of Complexity" (extended astract). In FOCS, pp. 222-227, 1977.

Christian Borgs, Microsoft Research New England, 1 Memorial Drive, Cambridge, MA 02142, USA (borgs@microsoft.com)

Michael Brautbar, Computer and Information Science Department, University of Pennsylvania, Philadelphia, PA, USA. Current address: Department of Electrical Engineering and Computer Science, Massachusetts Institute of Technology, 77 Massachusetts Avenue, Cambridge, MA 02139, USA (mbrautbar@gmail.com)

Jennifer Chayes, Microsoft Research New England, 1 Memorial Drive, Cambridge, MA 02142, USA (jchayes@microsoft.com)

Shang-Hua Teng, Computer Science Department, University of Southern California, 941 Bloom Walk, Los Angeles, CA 90089, USA (shanghua@usc.edu) 